1 Microbiota Accessible Carbohydrates Suppress Clostridium difficile Infection in a

2 Murine Model

3

4 Andrew J Hryckowian ${ }^{1}$, William Van Treuren ${ }^{1}$, Samuel A Smits ${ }^{1}$, Nicole M Davis ${ }^{1}$,

5 Jackson O Gardner ${ }^{1}$, Donna M Bouley ${ }^{2}$, Justin L Sonnenburg ${ }^{1 *}$

6

$7{ }^{1}$ Stanford University School of Medicine, Department of Microbiology and Immunology,

8 Stanford, CA; ${ }^{2}$ Stanford University School of Medicine, Department of Comparative

9 Medicine, Stanford, CA

10

$11{ }^{*}$ Corresponding Author, jsonnenburg@stanford.edu

12

13

14

15

16

17

18

19

20

21

22

23

24

25

26

27

28

29

30

31

32

33

34

35

36

37

38

39

40 
42 (CDI) represents a major healthcare concern, causing an estimated 15,000 deaths per

43 year in the United States alone ${ }^{1}$. Several enteric pathogens, including $C d$, leverage

44 inflammation and the accompanying microbial dysbiosis to thrive in the distal gut ${ }^{2}$.

45 Although diet is among the most powerful available tools for affecting the health of

46 humans and their relationship with their microbiota, investigation into the effects of diet

47 on CDI has been limited. Here, we show in mice that the consumption of microbiota

48 accessible carbohydrates (MACs) found in dietary plant polysaccharides has a

49 significant impact on CDI. Specifically, using a model of antibiotic-induced CDI that

50 typically resolves within 12 days of infection, we demonstrate that MAC-deficient diets

51 perpetuate CDI. We show that $C d$ burdens are suppressed through the addition of either

52 a diet containing a complex mixture of MACs or a simplified diet containing inulin as the

53 sole MAC source. We show that switches between these dietary conditions are

54 coincident with changes to microbiota membership, its metabolic output and Cd-

55 mediated inflammation. Together, our data demonstrate the outgrowth of MAC-utilizing

56 taxa and the associated end products of MAC metabolism, namely the short chain fatty

57 acids (SCFAs) acetate, propionate, and butyrate, are associated with decreased Cd

58 fitness despite increased $C d$ toxin expression in the gut. Our findings, when placed into

59 the context of the known fiber deficiencies of a human Western diet, provide rationale for

60 pursuing MAC-centric dietary strategies as an alternate line of investigation for mitigating

61 CDI.

62 CDI is typically associated with antibiotic-mediated dysbiosis, yet $22 \%$ of

63 individuals with community acquired CDI have no recent history of antibiotic use ${ }^{3}$. We

64 and others previously demonstrated that direct microbiota- $C d$ metabolic interactions are

65 critical determinants of $C d$ fitness in the distal gut ${ }^{4-6}$ and that the absence of dietary

66 MACs leads to the expression of inflammatory markers by the host colonic epithelium ${ }^{7}$. 
67 Additional in vitro work suggested that MAC-centric metabolic interactions may play a

68 role in reducing the fitness of $C d$ in the gut ${ }^{8-10}$, leading us to hypothesize that a MAC-

69 deficient diet reinforces the inflammation and dysbiosis conducive to CDI.

70 We used an experimental model of CDI in ex-germ-free Swiss-Webster mice

71 colonized with the microbiota of a healthy human donor (see Methods). These

72 humanized mice were fed a diet containing a complex mixture of MACs $\left(\mathrm{MAC}^{+}\right)$, or two

73 diets that are both MAC-deficient (MD) (Fig. 1a). Mice fed the MD diets show persistent

$74 \mathrm{CDI}$ while mice fed the $\mathrm{MAC}^{+}$diet clear the pathogen below detection within 10 days of

75 infection. After 36 days of persistent infection in mice fed the MD diets, a dietary shift to

76 the $\mathrm{MAC}^{+}$diet results in clearance below detection within 9 days (Fig. 1a). This $\mathrm{MAC}^{+}$

77 diet-mediated CDI suppression is also observed in conventional C57BL/6 and Swiss-

78 Webster mice and in ex-germ-free Swiss-Webster mice colonized with a conventional

79 Swiss-Webster mouse microbiota (Fig. S1), demonstrating that MAC-dependent CDI

80 suppression is not confined to a specific microbiota or host genotype.

81 To enumerate gut-resident microbes that might suppress $C d$, we sequenced $16 S$

82 rRNA amplicons from the feces of humanized mice (Fig. 1a). The presence of dietary

83 MACs and treatment with antibiotics affected both alpha and beta diversity of operational

84 taxonomic units (OTUs) in the gut microbiota (Figs. 1b-d, S2, S3). Two principle

85 coordinates explain $48 \%$ of the variance in weighted UniFrac distances between

86 samples. We traced temporal changes in the composition of the microbiota through this

87 space. To highlight the composition of the microbiota in the context of CDI, a log-fold

88 contour plot was drawn to illustrate burdens of $C d$ that correspond to these samples (see

89 Fig. S4 for further explanation of these "contoured PCoA" [cPCoA] plots).

90 Clindamycin remodels the mouse microbiome to a $C d$-permissive state (Figs.

91 1b-d, dotted lines; Fig. S3). After inoculation with $C d$, the microbiota of $\mathrm{MAC}^{+}$-fed mice

92 changes significantly, and as $C d$ burdens decrease, the community returns to resemble 
93 the pre-infection state (Figs. 1b, S3a), illustrating compositional resilience of the

94 microbiota under the $\mathrm{MAC}^{+}$dietary condition during CDI. During persistent infection in

95 mice fed the MD diets, the microbiota composition is similar to the pre-infection MAC-

96 deficient-associated microbiota. However, after the $\mathrm{MAC}^{+}$dietary intervention, the

97 microbiota of these mice transitions to resemble the microbiota of mice prophylactically

98 fed the $\mathrm{MAC}^{+}$diet as $\mathrm{Cd}$ burdens decrease (Figs. 1c, 1d, S3b, S3c). These data

99 suggest that diet and antibiotic treatment are two major drivers of microbial communities

100 that support or exclude $C d$ in our model. Furthermore, the similarities in the microbiota of

101 uninfected and persistently infected mice fed the MD diets may be due to the metabolic

102 and compositional constraints imposed by this dietary condition, which we hypothesize is

103 supportive to Cdduring infection.

104 Having shown that the $\mathrm{MAC}^{+}$diet, containing a complex and ill-defined mixture of

105 MACs (see Methods), is successful in suppressing CDI, we sought to decouple the

106 effects of MACs from other dietary components (e.g. phytonutrient ${ }^{11}$ or protein $^{12}$

107 content). Using a simplified MD1-based diet containing inulin as the sole MAC source,

108 the findings from our initial dietary intervention experiment in $\mathrm{Cd}$-infected animals are

109 recapitulated (Figs. 2a, S5, S6, Supplemental Text 1). Furthermore, prophylactic inulin

110 feeding (either $10 \%$ in the diet as above or $1 \%$ in the drinking water of mice fed the MD1

111 diet), results in dose-dependent effects on both the maximum $\mathrm{Cd}$ burden and on $\mathrm{Cd}$

112 clearance kinetics (Fig. S7). Taken together, like the complex $\mathrm{MAC}^{+}$diet, inulin feeding

113 reduces $C d$ burdens across experimental paradigms (Figs. 2a, S6-S8, Supplemental

\section{Text 2).}

115 Although the complex $\mathrm{MAC}^{+}$and the inulin-containing diets both negatively

116 impact the in vivo fitness of $C d$, the overall microbiome composition differs between mice

117 fed these two diets, as illustrated by a two-dimensional cPCoA subspace that explains

$11862 \%$ of the variation in the data and the proportional abundance of taxa (Figs. $\mathbf{2 b}$, S9, 
119 S10). Because increased gut microbiota diversity is associated with resistance to a

120 number of pathogens and is a hallmark of FMT-mediated suppression of $\mathrm{CDI}^{6,13-15}$, one

121 hypothesis is that MACs positively affect CDI outcome by favoring a diverse microbiota.

122 Though CDI suppression induced by the $\mathrm{MAC}^{+}$diet is correlated with an increase in

123 alpha diversity of the gut microbiota, alpha diversity does not increase when Cd burdens

124 decrease via inulin feeding (Figs. 2c, S11a). Furthermore, community evenness is

125 lowest in the inulin fed mice (Fig. S11b), consistent with a limited number of taxa

126 profiting from a single MAC type ${ }^{16}$. Together, these data demonstrate that MACs

127 significantly reduce $C d$ burdens but that this reduction is independent of microbiota

128 diversity.

129 Despite these wholesale differences in community composition, we pursued two

130 of several possibilities: (1) a common subset of OTUs suppress CDI across dietary

131 interventions or (2) diet-specific but functionally similar OTUs within dietary conditions

132 suppress CDI (see Supplemental Text 3, Tables S1, S2). Notably, several taxa are

133 significantly (anti)correlated with Cd burdens regardless of diet, suggesting that common

134 microbial signatures underlie permissive and non-permissive states (Table S3). Among

135 these, Parabacteroides, Lachnospiraceae, and Erysipelotrichaceae are correlated with

136 Cd abundance regardless of diet. The correlation between Parabacteroides and Cd

137 burden is consistent with previous observations that Parabacteroides are elevated in Cd

138 "supershedder" mice ${ }^{17}$. In humans, Erysipelotrichaceae and some Lachnospiraceae are

139 enriched in individuals with CDI compared to nondiarrheal controls ${ }^{18}$. Despite these

140 commonalities, the majority of features identified in Table S3 are (anti)correlated with Cd

141 in a subset of diets, supporting previous work that distinct context-dependent

142 communities, rather than core $C d$-(un)supportive communities, are important for

143 determining CDI status ${ }^{19}$. Because others have demonstrated that metabolites, rather 
144 than microbes, are able to differentiate CDI status in humans ${ }^{20}$, we hypothesized that

145 diet creates metabolic landscapes that are either supportive or unsupportive of $C d$.

146 Therefore, we pursued whether MAC-mediated suppression of CDI could be

147 differentiated from the permissive condition on a molecular basis relevant to MAC

148 metabolism. We measured the major metabolic end products of MAC metabolism (the

149 SCFAs acetate, propionate, and butyrate) in cecal contents of mice (see Fig S6).

150 Acetate and butyrate are elevated in the ceca of mice fed $\mathrm{MAC}^{+}$and inulin diets relative

151 to those fed the MD1 diet, and propionate is elevated in the ceca of $\mathrm{MAC}^{+}$-fed mice

152 relative to those fed the MD1 or inulin diets (Fig. 3a). Furthermore, acetate, propionate,

153 and butyrate have concentration-dependent negative effects on $\mathrm{Cd}$ growth as measured

154 by differences in doubling time (Fig. 3b) and concentration-dependent positive effects on

155 expression of a critical $C d$ virulence factor, the glycosylating toxin $\operatorname{TcdB}^{21}$ (Fig. 3c). Our

156 findings using $C d$ strain 630 are consistent with previous findings in other $C d$ strains: $(i)$

157 SCFAs inhibit the growth of five non-630 strains in a concentration dependent

158 fashion $^{10,22}$ and (ii) butyrate affects toxin expression in Cd strain VPI $10463^{23}$.

159 Given these findings, we hypothesize that dietary MACs negatively affect the

160 fitness of $C d$ in two interrelated ways. First, MACs drive privileged outgrowth of MAC-

161 utilizing members of the microbiota (e.g. Bacteroides spp., see Table S2b). Second, the

162 SCFAs that result from MAC metabolism negatively affect the fitness of $C d$, which could

163 be due to the buildup of endproducts of key metabolic pathways, such as reductive

164 acetogenesis and butyrogenesis ${ }^{5,24}$. The expression of TcdB (and the co-regulated toxin

$165 \mathrm{TcdA}$ ) in $C d$ is controlled by multiple inputs, such as nutrient availability, quorum

166 sensing, and other environmental stresses ${ }^{25}$. Therefore, it is possible that SCFAs serve

167 as a signal to $C d$ of microbiome fermentation, and this signal of a competitive and

168 inhospitable gut environment leads to an increase in toxin production. 
Since limitation of dietary MACs is known to increase inflammation in the

170 gastrointestinal tract ${ }^{7}$, we examined colonic histopathology and toxin levels to better

171 understand the inflammation during MAC-dependent suppression of CDI. Humanized

172 Swiss-Webster mice fed the MD1 diet were infected as in Fig. 1a and were switched to

173 either the $\mathrm{MAC}^{+}$or inulin diet at 7 days post infection. At pre- and post-diet shift time

174 points, histopathology of proximal colon tissue was evaluated. Pathology was

175 significantly increased in all infected mice relative to uninfected control mice fed the

$176 \mathrm{MAC}^{+}$or inulin diets (Figs. 4a, S12; Table S4). Notably, inflammation is comparably

177 elevated in both infected and uninfected mice fed the MD1 diet, consistent with the

178 contribution of the MD diets to inflammation and $\mathrm{Cd}$ persistence.

179 We also measured burdens of $C d$ and levels of TcdB in feces during the shift

180 from the MD1 diet to either the $\mathrm{MAC}^{+}$or inulin diets. TcdB is detected during persistent

181 infection but its expression is further elevated at 2 and 4 days after the shift to the $\mathrm{MAC}^{+}$

182 or inulin diet (Fig. 4b). Though cfu-normalized TcdB abundance is elevated after the diet

183 shift, the overall abundance of TcdB decreases from day 2 to day 4 post diet shift (Fig.

184 S13). This shows that toxin expression is elevated on a per-cell basis in response to

185 MACs but that overall TcdB abundance scales with decreasing burdens of $C d$. Together,

186 these data support a model where MAC deficient diets facilitate a level of inflammation

187 supportive of $C d$ survival in the gut, enabling $C d$ persistence despite lower levels of toxin

188 expression. $C d$ responds to the non-permissive MAC-driven environmental change by

189 elevating toxin expression. However, $\mathrm{Cd}$ is unable to maintain or regain its niche in the

190 gastrointestinal tract upon the sustained consumption of MACs by the host.

191 In the context of CDI, the relative contribution of inflammation to the exclusion of

192 inflammation-sensitive competitors versus the creation of privileged nutrients, analogous

193 to strategies delineated for the enteric pathogen Salmonella Typhimurium, remains to be

194 determined ${ }^{2}$. Furthermore, though SCFAs can directly inhibit bacterial growth and affect 
195 Cd toxin production, additional work is needed to investigate the potential roles of other

196 relevant factors that could be impacting infection dynamics. For example, SCFA can

197 signal through host pathways (e.g. improved barrier function via hypoxia-inducible

198 factor $^{26}$ ) which may influence inflammation and microbiome composition independently

199 of $C d$ burdens and toxin production; other metabolites such as bile acids can influence

$200 \mathrm{CDI}^{6}$ and may interact with dietary effects.

201 Current microbiota-centric therapies for CDI, such as fecal microbiota transplant

202 and probiotic administration, focus on the introduction of exogenous organisms. Our

203 work in mice shows that dietary intervention supports microbial communities that

204 exclude $C d$ without the requirement for microbe introduction. Regardless of the inter-

205 experiment and inter-animal variations in clearance kinetics (Fig. S8), the effect is highly

206 reproducible. Clearance kinetics may be further affected by host genetics, initial

207 microbiota composition, or overall dietary MAC concentration/composition.

208 Despite the individuality in the gut microbiota of patients with CDI, there is a

209 consistent metabolic response that underlies CDI across individuals ${ }^{20}$. In light of

210 observations that MACs profoundly alter the composition and function of the microbiota

211 and host physiology ${ }^{27}$, our findings raise the possibility that SCFAs are a critical part of

212 the metabolic landscapes tied to CDI status across individuals. Currently, several

213 technical hurdles exist to experimentally manipulating SCFAs within the colon,

214 presenting a tremendous opportunity to develop new methods that enable exploring this

215 important class of fermentation end-products and their effects on gut ecology

216 (Supplemental Text 4).

217 Notably, two independent human trials have shown cooked green bananas (rich

218 in MACs as evident by elevated SCFAs in the stool of treated patients) aid host recovery

219 from another enteric pathogen, Shigella $a^{28,29}$. More recently, it was shown that a MAC-

220 deficient diet leads to microbiota-dependent mucus degradation and attachment- 
221 dependent lethal colitis by the murine pathogen, Citrobacter rodentium ${ }^{30}$. Taken

222 together, our work is part of a growing body of literature providing evidence that dietary

223 manipulation of the metabolic networks of the intestinal tract is a powerful and

224 underexplored way to influence gastrointestinal pathogens.

225

226 Methods

\section{Media and bacterial growth conditions}

228 Frozen stocks of Clostridium difficile $(C d)$ strain $630^{31}$ were maintained under

229 anaerobic conditions in septum-topped vials. Cd 630 was routinely cultured on CDMN

230 agar, composed of $C d$ agar base (Oxoid) supplemented with $7 \%$ defibrinated horse

231 blood (Lampire Biological Laboratories), $32 \mathrm{mg} / \mathrm{L}$ moxalactam (Santa Cruz

232 Biotechnology), and $12 \mathrm{mg} / \mathrm{L}$ norfloxacin (Sigma-Aldrich) in an anaerobic chamber at $37^{\circ}$

233 (Coy). After 16-24 hours of growth, a single colony was picked into $5 \mathrm{~mL}$ of pre-reduced

234 Reinforced Clostridial medium (RCM, Oxoid) and grown for 16 hours. This 16-hour

235 culture was used to inoculate mice, below.

236 For in vitro experiments, Cd 630 was cultured on CDMN as above. Single

237 colonies were picked into pre-reduced Cd minimal medium (CDMM) without glucose, as

238 described previously ${ }^{32}$. After 16 hours of growth, subcultures were prepared at a 1:200

239 dilution in pre-reduced CDMM supplemented with 0,10 , or $30 \mathrm{mM}$ of sodium acetate

240 (Fisher), sodium propionate (Sigma Aldrich), sodium butyrate (Sigma Aldrich), or sodium

241 chloride (EMD Millipore) in sterile polystyrene 96 well tissue culture plates with low

242 evaporation lids (Falcon). To further minimize evaporation of culture media during

243 growth, the 36 wells along the perimeter of the 96 well plates were filled with water

244 rather than culture. Cultures were grown anaerobically as above in a BioTek Powerwave

245 plate reader. At 15-minute intervals, the plate was shaken on the 'slow' setting for 1

246 minute and the optical density $\left(O D_{600}\right)$ of the cultures was recorded using Gen5 software 
247 (version 1.11.5). After 24 hours of growth, culture supernatants were collected after

248 centrifugation ( 5 minutes at $2,500 \times \mathrm{g}$ ) and stored at $-20^{\circ} \mathrm{C}$ for quantification of $\mathrm{TcdB}$ (see

249 Quantification of C. difficile toxin TcdB, below).

250

\section{Murine model CDI}

$252 \quad$ All animal studies were conducted in strict accordance with the Stanford

253 University Institutional Animal Care and Use Committee (IACUC) guidelines. Murine

254 model CDI was performed on age- and sex-matched mice between 8 and 17 weeks of

255 age, possessing one of three gut microbiota colonization states: (1) Humanized Swiss-

256 Webster mice: Germ free mice (SWGF, Taconic; bred in house) were inoculated with a

257 fecal sample obtained from a healthy anonymous donor, as used in previous studies

258 from our laboratory ${ }^{33,34}$, (2) Conventionally-reared Swiss-Webster mice (SWRF, Taconic;

259 bred in house); or C57BL/6 mice (B6EF, Taconic; experiments conducted on animals

260 acquired directly from vendor), and (3) Conventionalized mice: Germ free Swiss-

261 Webster mice were inoculated with a fecal sample obtained from SWRF mice. The gut

262 microbiomes of the humanized and conventionalized mice were allowed to engraft for at

263 least 4 weeks ${ }^{35}$.

264 To initiate CDI, mice were given a single dose of clindamycin by oral gavage (1

$265 \mathrm{mg} ; 200 \mu \mathrm{L}$ of a $5 \mathrm{mg} / \mathrm{mL}$ solution) and were infected 24 hours later with $200 \mu \mathrm{L}$ of

266 overnight culture grown in RCM (approximately $1.5 \times 10^{7} \mathrm{cfu} / \mathrm{mL}$ ) or mock infected with

$267200 \mu \mathrm{L}$ filter sterilized PBS. To reactivate CDI in mice that had cleared the infection

268 below detection, mice were given a single dose of clindamycin as above.

269 Feces were collected from mice directly into microcentrifuge tubes and placed on

270 ice. To monitor $\mathrm{Cd}$ burdens in feces, $1 \mu \mathrm{L}$ of each fecal sample was resuspended in PBS

271 to a final volume of $200 \mu \mathrm{L}, 10$-fold serial dilutions of fecal slurries (through $10^{-3}$-fold)

272 were prepared in sterile polystyrene 96 well tissue culture plates (Falcon). For each 
273 sample, duplicate $10 \mu \mathrm{L}$ aliquots of each dilution were spread onto CDMN agar. After 16-

27424 hours of anaerobic growth at $37^{\circ} \mathrm{C}$, colonies were enumerated and duplicate spots

275 were averaged to give cfu values (limit of detection $=2 \times 10^{4} \mathrm{cfu} / \mathrm{mL}$ feces). $\mathrm{Cd}$ was

276 undetectable in all mice prior to inoculation with Cd (Figs. 1A, S1, S6-S8) and in all mice

277 that were mock infected with PBS (Fig. S8), supporting that the animals used in this

278 work were not pre-colonized with $C d$ (e.g. Cd LEM1, as seen by Etienne-Mesmin and

279 colleagues $\left.^{36}\right)$. After serial dilution of fecal samples, the remaining amounts of fecal

280 samples were immediately frozen at $-80^{\circ} \mathrm{C}$ until needed for $16 \mathrm{~S}$ rRNA analysis and TcdB

281 ELISAs, below. It was not possible to blind researchers to infection or dietary status of

282 the animals.

283

\section{$284 \quad$ Mouse diets}

Mice were fed one of four diets in this study ad libitum: (1) a diet containing a complex mixture of MACs (MAC ${ }^{+}$, Purina LabDiet 5010); (2) a custom MAC-deficient

$287 \operatorname{diet}^{37}[\mathrm{MD} 1,68 \%$ glucose $(\mathrm{w} / \mathrm{v}), 18 \%$ protein $(\mathrm{w} / \mathrm{v})$, and 7\% fat (w/v) (Bio-Serv); (3) a 288 commercially available MAC-deficient diet [MD2, 34\% sucrose (w/v), 17\% protein (w/v), $28921 \%$ fat (w/v); Harlan TD.88137], or (4) a custom diet containing inulin as the sole MAC 290 source $^{37}$, which is based on the MD1 diet [58\% glucose (w/v); $10 \%$ inulin $(w / v)[$ Beneo-

291 Orafti group; OraftiHP]; 18\% protein (w/v), and 7\% fat (w/v) (Bio-Serv)]. Where

292 applicable (see Fig. S7), the drinking water of mice fed the MD1 diet was supplemented 293 with 1\% inulin (w/v) [Beneo-Orafti group; OraftiHP]. Because mice consume

294 approximately 5 grams of food per day and $5 \mathrm{~mL}$ of water per day ${ }^{38}$, water with $1 \%$ inulin 295 gives an approximate 10 -fold reduction in inulin consumed relative to the $10 \%$ inulin diet.

296 Where applicable (see Fig. S14), mice fed the MD1 diet were gavaged daily with $200 \mu \mathrm{L}$ 297 tributyrin (Sigma Aldrich) or were given a cocktail of sodium acetate (67.5 mM, Fisher), 298 sodium propionate ( $25 \mathrm{mM}$, Sigma Aldrich), and sodium butyrate (40 mM, Sigma 
299 Aldrich) in their drinking water ${ }^{39}$ starting 1 day pre-infection. Groups of mice were

300 randomly assigned to dietary conditions.

\section{Histology and histopathological scoring}

303 Proximal colon sections harvested for histopathologic analyses were fixed in 10\%

304 buffered neutral formalin and routinely processed for paraffin embedding, sectioned at 4

305 microns, mounted on glass slides and stained with hematoxylin and eosin (Histo-tec

306 Laboratory, Hayward, CA). Analyses were performed by a board certified veterinary

307 pathologist, using a semiquantitative scoring system ${ }^{40}$ that evaluated distribution and

308 severity of cellular infiltrates (inflammation), gland hyperplasia, edema, and epithelial

309 disruption using a severity score of 0 to $5(0=$ no significant lesion, $1=$ minimal, $2=$ mild,

3103 = moderate, 4 = marked, 5 = severe, see Table S4).

311

\section{Quantification of C. difficile toxin TcdB}

313 Levels of TcdB in culture supernatants and feces were quantified relative to a

314 standard curve of purified TcdB using the "Separate detection of $C$. difficile toxins A and

315 B" kit (TGC Biomics) according to the instructions that are readily available on the

316 manufacturer's website. For culture supernatants which were harvested at 24 hours

317 post-inoculation (see Media and bacterial growth conditions, above), toxin abundance

318 was normalized by the final $\mathrm{OD}_{600}$ of the culture and "Normalized TcdB Abundance in

319 Culture Supernatant" $=[(x$ ng toxin $) /(y$ OD600) $)$ and is reported in Fig. 3c. For each

320 fecal sample, toxin abundance was normalized by the number colony forming units, as

321 determined by selective culture on CDMN agar (above), for each sample; "Normalized

322 TcdB Abundance in Feces" = [(x ng toxin gram $^{-1}$ feces $) /\left(y\right.$ cfu $C$. difficile $\mathrm{mL}^{-1}$ feces $\left.)\right]$

323 and is reported in Fig. 4b. Non cfu-normalized TcdB abundance (ng toxin gram $^{-1}$ feces)

324 in mouse feces is reported in Fig. S13. 


\section{16S rRNA amplicon sequencing and OTU picking methods}

327 Total DNA was extracted from frozen fecal material using the PowerSoil DNA

328 Isolation Kit (MoBio) or the Powersoil-htp 96 well DNA isolation kit (MoBio). Barcoded

329 primers were used to amplify the V3-V4 region of the 16S rRNA gene from extracted

330 bacterial DNA using primers $515 f$ and $806 \mathrm{rB}$ via $\mathrm{PCR}^{41}$. Amplicon cleanup was

331 performed using the UltraClean PCR Clean-Up Kit (MoBio) and quantification was

332 performed using the high sensitivity Quant-iT dsDNA Assay Kit (Thermo Fisher).

333 Amplicons were pooled to an equimolar ratio. Amplicons from 3 mouse experiments

334 were sequenced in 3 different paired-end Illumina MiSeq runs, with each experiment

335 occurring on a separate run. The sample split/run corresponds to the field 'Experiment'

336 in Table S1.

337 For commands executed for the 16S rRNA-based bioinformatics analysis, please

338 see Code S1, an ipython notebook. Runs were demultiplexed independently due to

339 some non-unique barcodes, and then concatenated prior to OTU picking using

340 'split_libraries_fastq.py' with default quality parameters in QIIME 1.9.1 ${ }^{42}$. Open reference

341 OTU picking was conducted with default parameters using the QIIME script

342 'pick_open_reference_otus.py' (with default clustering algorithm UCLUST ${ }^{43}$ ) on the

$34324,582,127$ reads that passed quality filtering. OTUs whose representative sequence

344 failed to align to the Greengenes reference alignment with at $85 \%$ identity using PyNAST

345 were discarded $^{44,45}$.

346 We removed OTUs occurring in at least 10 samples, and/or having less than 26

347 counts in the entire dataset. This filtering reduced the number of OTUs by $95.04 \%$

$348(211,884$ to 10,504$)$ but removed $5.2 \%$ of the feature-mass $(23,293,178$ to $22,078,743)$.

349 This type of filtering removes a vast number of features that are likely artefacts, boosts

350 power by reducing false discovery penalties, and concentrates analysis on biologically 
351 meaningful features. We rarefied our data to correct for differences sequencing depth.

352 To ensure our results were not artefacts of rarefaction depth we conducted analyses at

353 multiple rarefaction levels and our conclusions were not changed. We use OTU tables

354 rarefied to 7,000 in this study, facilitating inter-run comparisons.

\section{Supervised learning}

357 Using the 'supervised_learning.py' script from QIIME 1.9.1, the random forests

358 classification method (with 10-fold cross validation error estimation) was trained using an

359 OTU table as prepared above in "16S sequencing and OTU picking methods." Presence

360 or absence of $C d$ in a fecal sample (as determined by selective culture) or current diet

361 were used as the class label category, corresponding to the field 'Plus_minus_Cd' and

362 'Current_diet' of Table S1. The OTU table was modified for this analysis by querying

363 each of the 11 Cd 630 rRNA sequences against a BLAST database built from the

364 representative set of OTUs created during OTU picking (see "16S sequencing and OTU

365 picking methods"), after which the OTUs that matched Cd 630 rRNA sequences (cutoff

$36697 \%$ identity) were collapsed into a single CdOTU,

367 “k_Bacteria;p__Frimicutes;c__Clostridia;o__Clostridiales;f__Peptostreptococcaceae;g_

368 _Clostridioides;s_putative_difficile." See Code S1 for the code used for this analysis.

\section{Quantification of short chain fatty acids (SCFAs)}

371 Immediately following euthanasia at 32 days post infection, cecal contents were

372 removed from mice described in Fig. S6, weighed, and flash frozen in liquid nitrogen.

373 Cecal contents $(70-150 \mathrm{mg})$ were suspended in a final volume of $600 \mu \mathrm{l}$ in ice-cold ultra

374 pure water and blended with a pellet pestle (Kimble Chase) on ice. The slurry was

375 centrifuged at $2,350 \times \mathrm{g}$ for 30 seconds at $4^{\circ} \mathrm{C}$ and $250 \mu \mathrm{L}$ of the supernatant was

376 removed to a septum-topped glass vial and acidified with $20 \mu \mathrm{L} \mathrm{HPLC}$ grade $37 \% \mathrm{HCl}$ 
377 (Sigma Aldrich). Diethyl ether $(500 \mu \mathrm{L})$ was added to the acidified cecal supernatant to

378 extract SCFAs. Samples were then vortexed at $4^{\circ} \mathrm{C}$ for 20 minutes on 'high' and then

379 were centrifuged at $1,000 \times \mathrm{g}$ for 3 minutes. The organic phase was removed into a fresh

380 septum-topped vial and placed on ice. Then, a second extraction was performed with

381 diethyl ether as above. The first and second extractions were combined for each sample

382 and $250 \mu \mathrm{L}$ of this combined solution was added to a $300 \mu \mathrm{L}$ glass insert in a fresh glass

383 septum-topped vial containing and the SCFAs were derivitized using $25 \mu \mathrm{L} \mathrm{N}$-tert-

384 butyldimethylsilyl-N-methyltrifluoroacetamide (MTBSTFA; Sigma Aldrich) at $60^{\circ} \mathrm{C}$ for 30

385 minutes.

386 Analyses were carried out using an Agilent 7890/5975 single quadrupole GC/MS

387 Using a 7683B autosampler, $1 \mu \mathrm{L}$ split injections (1:100) were made onto a DB-5MSUI

388 capillary column (30 m length, $0.25 \mathrm{~mm}$ ID, $0.25 \mu \mathrm{m}$ film thickness; Agilent) using helium

389 as the carrier gas $\left(1 \mathrm{~mL} /\right.$ minute, constant flow mode). Inlet temperature was $200^{\circ} \mathrm{C}$ and

390 transfer line temperature was $300^{\circ} \mathrm{C}$. GC temperature was held at $60^{\circ} \mathrm{C}$ for 2 minutes,

391 ramped at $40^{\circ} \mathrm{C} / \mathrm{min}$ to $160^{\circ} \mathrm{C}$, then ramped at $80^{\circ} / \mathrm{min}$ to $320^{\circ} \mathrm{C}$ and held for 2 minutes;

392 total run time was 8.5 minutes. The mass spectrometer used electron ionization $(70 \mathrm{eV})$

393 and scan range was $\mathrm{m} / \mathrm{z}$ 50-400, with a 3.75-minute solvent delay. Acetate, propionate,

394 and butyrate standards (20 mM, $2 \mathrm{mM}, 0.2 \mathrm{mM}, 0.02 \mathrm{mM}, 0 \mathrm{mM})$ were acidified,

395 extracted, and derivatized as above, were included in each run, and were used to

396 generate standard curves to enable SCFA quantification.

\section{Measurement of doubling time for in vitro growth experiments}

399 Raw $\mathrm{OD}_{600}$ measurements of cultures grown in CDMM (see "Media and bacterial

400 growth conditions," above) were exported from Gen5 to MATLAB and analyzed using

401 the growth_curve_analysis_v2_SCFA.m script and analyze_growth_curve_SCFA.m

402 function (Code S2 and Code S3, respectively). Growth rates were determined for each 
403 culture by calculating the derivative of natural log-transformed $\mathrm{OD}_{600}$ measurements over

404 time. Growth rate values at each time point were then smoothed using a moving average

405 over 75-minute intervals to minimize artefacts due to noise in OD measurement data. To

406 mitigate any remaining issues with noise in growth rate values, all growth rate curves

407 were also inspected manually. Specifically, in cases where the

408 analyze_growth_curve_SCFA function selected an artefactual maximum growth rate, the

409 largest local maximum that did not correspond to noise was manually assigned as the

410 maximum growth rate. Doubling time was then computed by dividing the natural log of 2

411 by maximum growth rate. The investigator that conducted growth curve analysis was

412 blinded to the experimental conditions in which growth curve data were obtained.

413

\section{Statistical methods}

$415 \quad$ Alpha and beta diversity, correlations, and random forests were computed using

416 QIIME ('alpha_diversity_through_plots.py', 'beta_diversity_through_plots.py',

417 'observation_metadata_correlations.py', 'supervised_learning.py'). Kruskal-Wallis,

418 Mann-Whitey, Student's T, ANOVA, and D'Agostino-Pearson tests were performed

419 using standard statistical analyses embedded in the Prism 7 software package

420 (GraphPad Software Inc.). Spearman correlations were calculated in Python using Code

421 S1 under the heading 'feature correlations by diet'. Specific statistical tests are noted in

422 figure legends or tables as applicable.

\section{Data availability}

425 The data that support the findings of this study are available from the corresponding 426 author upon request. The $16 \mathrm{~S}$ sequence data is uploaded to Qiita (http://qiita.ucsd.edu;

427 Study ID 11347).

428 


\section{Code availability}

430 For custom code used in this study, see Code S1-S3.

431

\section{Correspondence}

433 Please address all correspondence to Justin Sonnenburg (jsonnenburg@stanford.edu).

\section{Acknowledgements}

436 We thank Steven K. Higginbottom (Department of Microbiology and Immunology,

437 Stanford) for expertise and technical assistance in all mouse experiments, Allis S. Chien

438 (Stanford University Mass Spectrometry Facility) for developing the GC-MS parameters

439 used in this study, and Carlos G. Gonzalez (Department of Chemical and Systems

440 Biology, Stanford) for assistance with DNA extractions from mouse feces. This work was

441 funded by a grant from National Institutes of Health NIDDK (R01-DK085025 to J.L.S.),

442 an NIH postdoctoral NRSA (5T32AI007328 to A.J.H.), a Stanford University School of

443 Medicine Dean's Postdoctoral Fellowship (A.J.H.), NSF Graduate Research Fellowships

444 (S.A.S, W.V.T - DGE-114747), an NIH predoctoral NRSA (5T32AI007328 to N.M.D.),

445 and a Smith Stanford Graduate Fellowship (S.A.S.). J.L.S. received an Investigators in

446 the Pathogenesis of Infectious Disease Award from the Burroughs Wellcome Fund.

\section{Author Contributions}

449 A.J.H., N.M.D., and J.O.G. performed the experiments. D.M.B. conducted blinded

450 scoring, imaging, and analysis of tissue sections. A.J.H., W.V.T., S.A.S., N.M.D.,

451 D.M.B., and J.L.S. analyzed and interpreted data, designed experiments, and prepared

452 display items. A.J.H. and J.L.S. wrote the paper. All authors edited the manuscript prior

453 to submission. 


\section{Competing interests}

456 The authors declare no competing interests.

457

458

459

460

461

462

463

464

465

466

467

468 
469

470

471

472

473

474

475

476

477

478

479

480

481

482 a
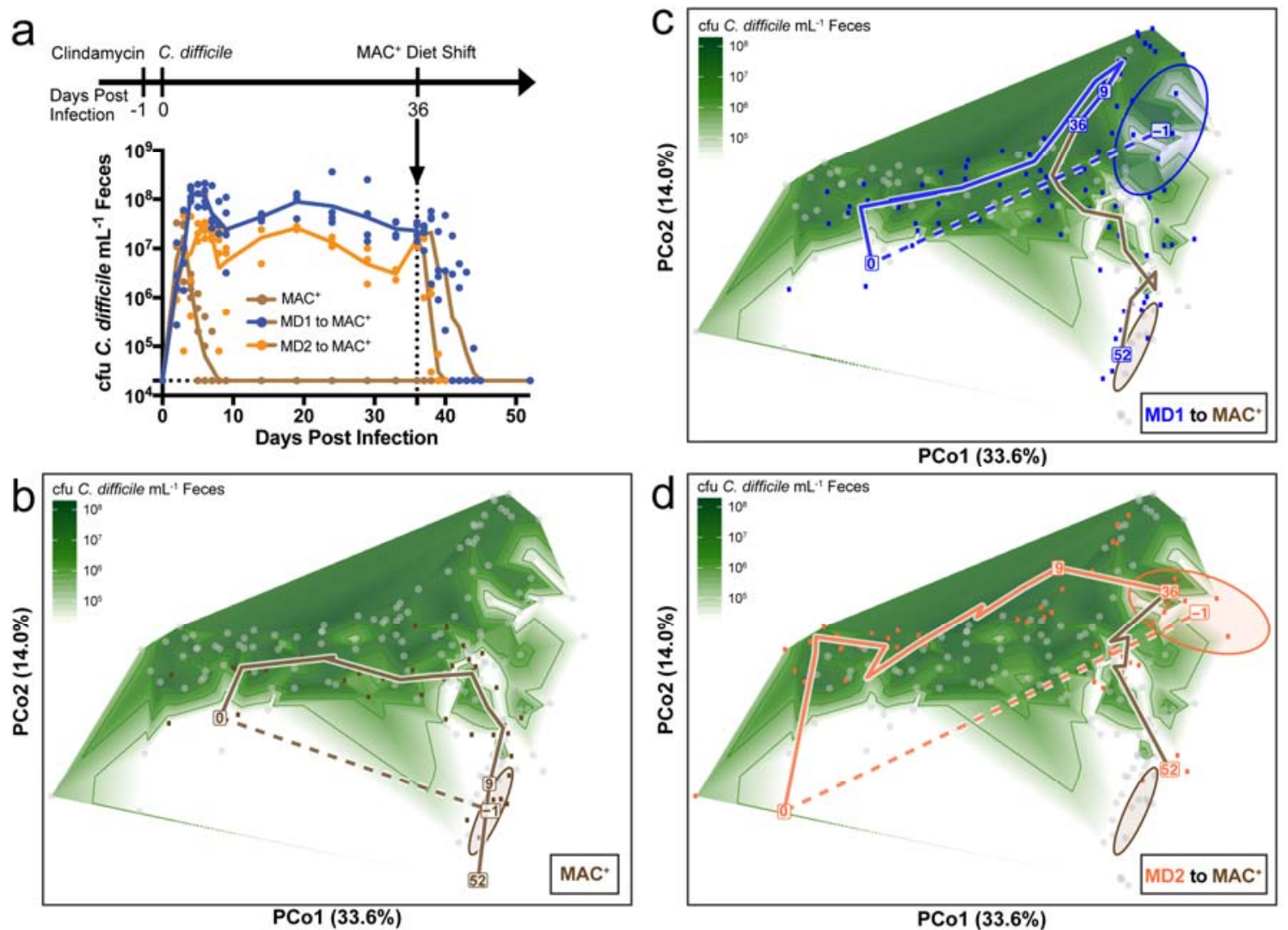

Figure 1. Dietary MACs toggle the fitness of Clostridium difficile $(C d)$ in the gut

while engendering distinct microbiota states. Humanized, age matched female

Swiss-Webster mice were maintained on a diet containing a complex mixture of MACs

(MAC $+n=4$ mice) or on diets deficient in MACs (MD1, $n=4$ mice; and MD2, $n=4$ mice)

starting 8 days pre-infection. See Methods for details of diet compositions. (a) Mice were subsequently gavaged with clindamycin at 1 day before infection with $C d$. At 36 days post-infection, mice fed the MD diets were switched to the $\mathrm{MAC}^{+}$diet. $\mathrm{Cd}$ burdens were monitored over time by selective culture, as described in Methods. One of the MD2 fed mice was moribund on D10 and was euthanized. Individual per-sample $C d$ burdens are plotted and lines represent geometric mean burdens per time point. (b-d) Principal coordinates analysis plots of Weighted UniFrac distances between microbiota samples collected from these mice were prepared and overlaid with log-fold contour plots of $C d$ burdens, as measured in panel a. Clindamycin affects the composition of the microbiota 
483 in all groups of mice from D-1 (annotated with ellipses representing $80 \% \mathrm{Cl}$ ) to D0

484 (dotted lines), resulting in a dysbiotic state permissive to CDI. In each panel, a line is

485 drawn through the centroid of the points for a given experiment day. (c) In mice fed the

$486 \mathrm{MAC}^{+}$diet, the microbiota returns to resemble the pre-infection state as Cd burdens

487 decrease. (d, e) In mice fed the MD1 and MD2 diets, respectively, CDI remains

488 unresolved until dietary intervention with the $\mathrm{MAC}^{+}$diet at $\mathrm{D} 36$, which shifts the

489 microbiota to resemble that of other $\mathrm{MAC}^{+}$fed mice as $\mathrm{Cd}$ burdens decrease (brown

490 lines). Points are colored by the highlighted treatment group, or alternatively are retained

491 as gray points for reference. See Fig. S4 for further explanation on generation and

492 interpretation of the contoured PCoA (cPCoA) plots shown in panels b-d.

493

494

495

496

497

498

500 
a

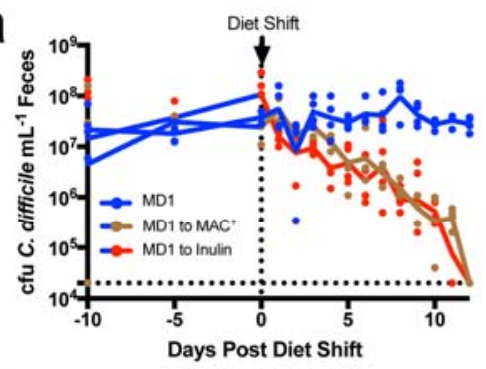

C

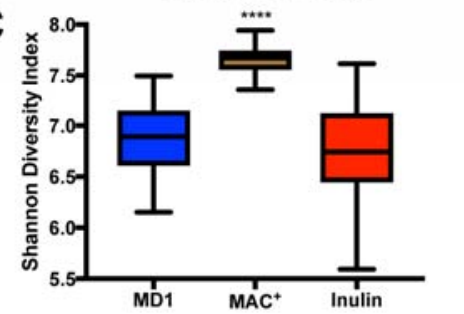

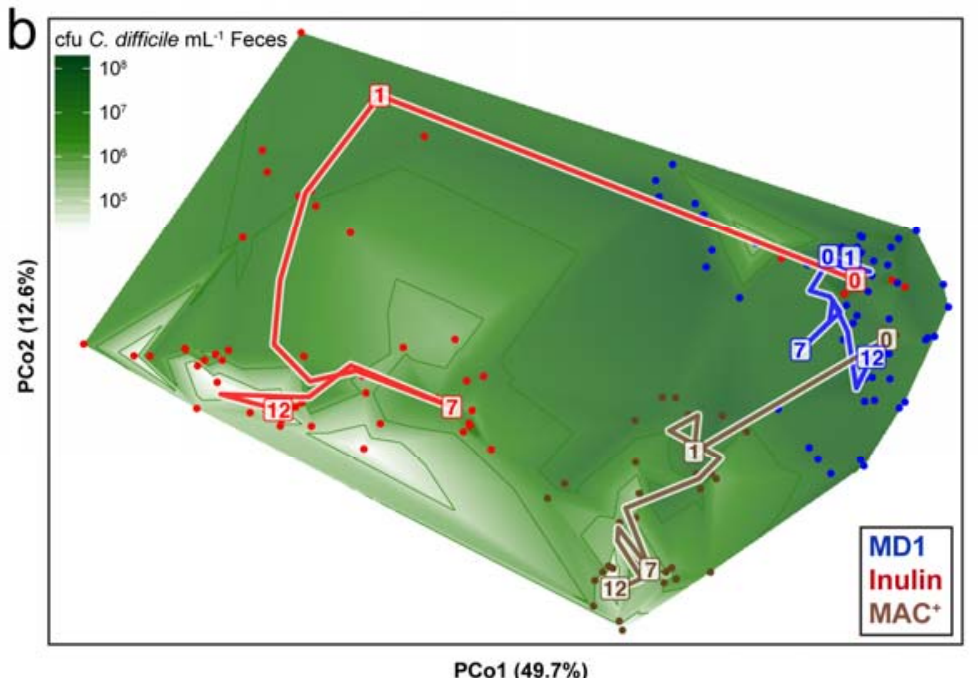

PCo1 $(49.7 \%)$

502 Figure 2. A diet containing inulin as the sole MAC source reduces $\mathrm{Cd}$ burdens

503 without increasing microbial diversity. Mice with persistent CDI were subjected to a

504 dietary intervention of the complex $\mathrm{MAC}^{+}$diet $(\mathrm{n}=3)$, a diet containing inulin as the sole

505 MAC source $(n=4)$, or were maintained on the MD1 diet $(n=4)$. See Methods for details

506 of diet compositions. (a) Burdens of $C d$ were monitored over time by selective culture as

507 described in Methods. Individual per-sample Cd burdens are plotted and lines represent

508 geometric mean burdens per time point. (b) A contoured PCoA (cPCoA) plot of weighted

509 UniFrac distances between microbiomes of these mice was prepared and overlaid with

510 log-fold contour plots of $C d$ burdens, as measured in panel A. A line is drawn through

511 the centroid of the points for a given experimental day. See Fig. S4 for further

512 explanation on generation and interpretation of $\mathrm{CPCoA}$ plots. (c) Alpha diversity of

513 communities was determined longitudinally for the microbiota of these mice by Shannon

514 Diversity Index. Differences in Cd burdens and alpha diversity between dietary

515 conditions was determined by collapsing all time points after the diet shift time point (See

516 Fig. $\mathbf{S 1 1}$ for temporal differences in Shannon Diversity Index). Statistical significance

517 was determined by Kruskal Wallis test with Dunn's multiple comparison test $\left(^{* * * *}=\right.$

$518 \quad \mathrm{p}<0.0001)$. 


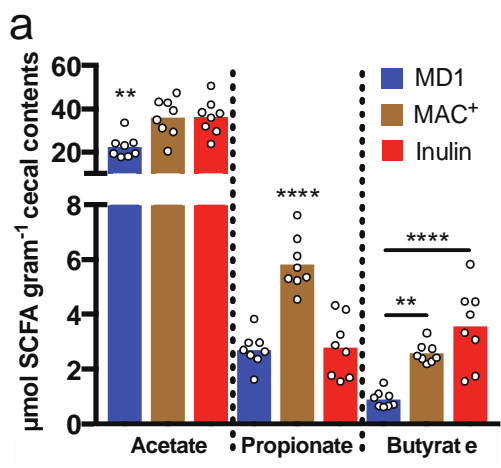

520

521 Figure 3. Acetate, propionate, and butyrate are elevated in the ceca of mice fed

522 MACs, and affect growth and toxin production in C. difficile. (a) The short chain

523 fatty acids acetate, propionate, and butyrate were measured in cecal contents of mice

524 fed the MD1, MAC ${ }^{+}$, and inulin containing diets ( $\mathrm{n}=8$ per dietary condition). See Fig S6

525 for time points of sacrifice corresponding to measurements). Data points represent per

526 sample concentration of each SCFA and bars represent the mean per-diet SCFA

527 concentration. Statistical significance was assessed by ordinary one-way ANOVA and

528 Tukey's multiple comparison test. ( $\left.{ }^{* \star}, p<0.01 ;{ }^{* * *}, p<0.0001\right)$ (b) Doubling time was

529 calculated for $C d$ grown in CDMM supplemented with 0, 10, and $30 \mathrm{mM}$ sodium acetate

530 (NaOAc), sodium propionate ( $\mathrm{NaOPr})$, sodium butyrate $(\mathrm{NaOBu})$, or sodium chloride

531 ( $\mathrm{NaCl}$, sodium matched controls) as described in Methods. For each growth condition, at

532 least $n=16$ independent cultures were assessed. (c) TcdB was quantified in

533 supernatants from $n=12$ independent cultures from each growth condition as described

534 in Methods. Points represent mean values, error bars represent \pm SEM (panels b-c).

535 Statistical significance was assessed for each SCFA and sodium matched controls at

536 each concentration via Mann-Whitney test (ns, not significant; ${ }^{*}, p<0.05 ;{ }^{* \star * *}, p<0.0001$ ). 


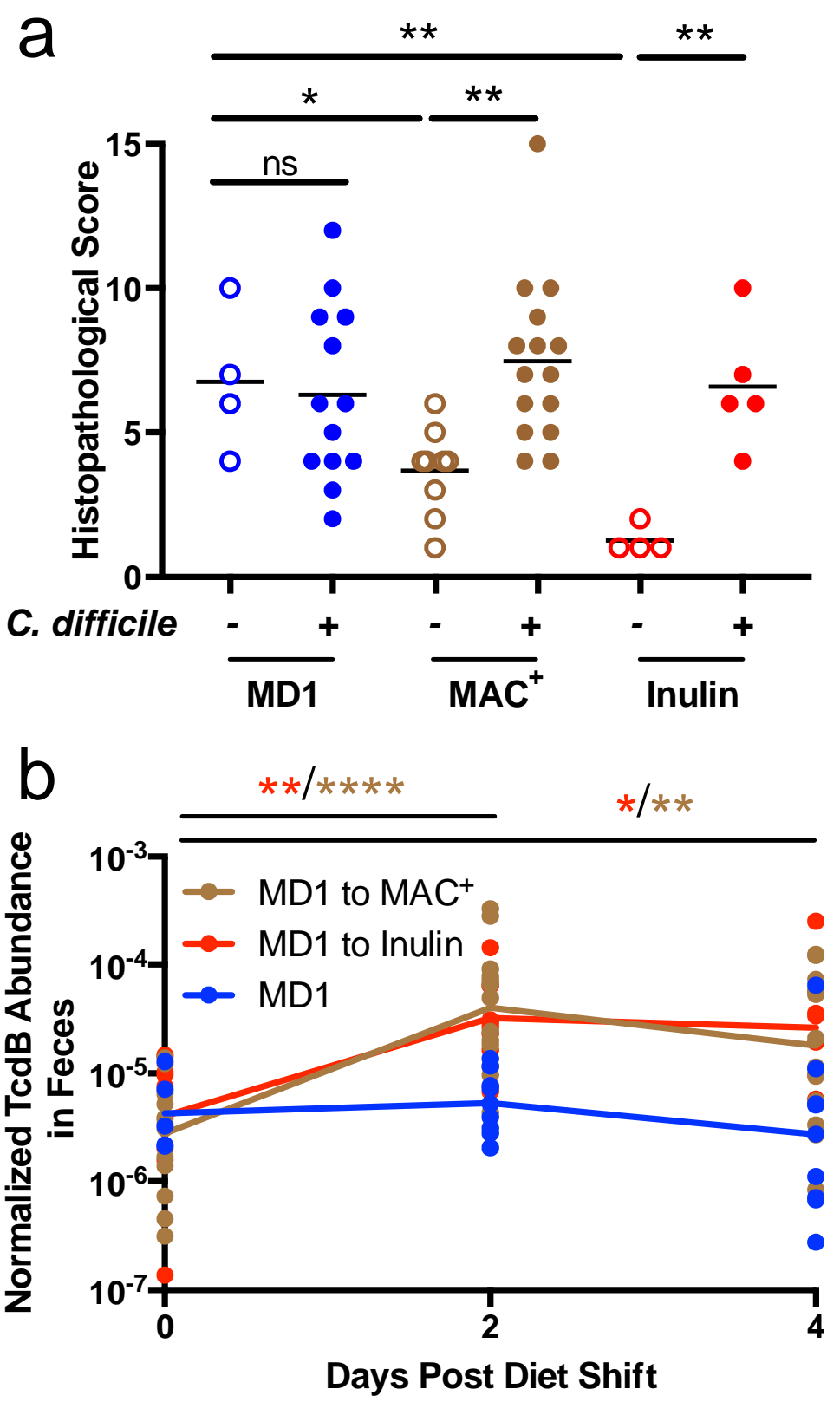

539 Figure 4. Inflammation and $\boldsymbol{C}$ d toxin expression are diet-dependent. Age-matched,

540 female, humanized Swiss-Webster mice were fed the MD1 diet, gavaged with

541 clindamycin, and subsequently infected with $C d$ as in Fig. 1 or were mock infected with

542 filter sterilized PBS. After 7 days of infection, mice were switched either to the $\mathrm{MAC}^{+}$or

543 inulin-containing diet. (a) Mice were euthanized before and after diet change, at time

544 points specified in Table S4. Histopathology was carried out on proximal colon tissue 
545 from these mice as described in Methods ( $n=4$ for mock-infected mice fed the MD1 diet,

$546 \mathrm{n}=9$ for mock-infected mice fed the $\mathrm{MAC}^{+}$diet, $\mathrm{n}=4$ for mock-infected mice fed the inulin

547 diet, $n=13$ for infected mice fed the MD1 diet, $n=15$ for infected mice fed the $\mathrm{MAC}^{+}$diet,

548 and $n=5$ for infected mice fed the inulin diet). Individual points represent the sum

549 histopathological score and lines are drawn at the mean score for each group. Statistical

550 significance between relevant groups was assessed by unpaired T test $\left({ }^{*}=p<0.05\right.$;

$\left.551{ }^{* *}=p<0.01\right)$. (b) For infected mice where $>5 \mathrm{mg}$ fecal material could be collected $(n=14$,

$552 \mathrm{n}=13$, and $\mathrm{n}=13$ for mice at 0,2 , and 4 days post MD1 to $M A C^{+}$diet shift; $n=8, n=9$, and

$553 \mathrm{n}=6$ for for mice at 0,2 , and 4 days post MD1 to inulin diet shift; and $n=5, n=9$, and $n=9$

554 at matched time points for mice that were maintained on the MD1 diet), levels of TcdB in

555 the feces were measured and normalized to the burdens of $C d$ detected. Points

556 represent normalized TcdB abundance for individual fecal samples lines are drawn

557 through the per-day per-diet geometric mean normalized toxin abundance. For mice

558 switched to the $\mathrm{MAC}^{+}$diet, normalized TcdB abundance increases 14.5-fold from day 0

559 to day $2(p<0.0001)$ and 6.5 -fold from day 0 to day $4(p=0.0066)$. For mice switched to

560 the inulin diet, normalized TcdB abundance increases 8.0-fold from day 0 to day 2

$561(p=0.0016)$ and 6.5-fold from day 0 to day $4(p=0.0426)$. Statistical significance between

562 relevant pairs of treatment groups was assessed by Mann-Whitney test $\left({ }^{*}=p<0.05 ;{ }^{* *}=\right.$

$\left.563 \mathrm{p}<0.01 ;{ }^{* * *}=\mathrm{p}<0.001 ;{ }^{* * *}=\mathrm{p}<0.0001\right)$.

564

565

566

567

568

569

570 


\section{References}

5721 Lessa, F. C., Winston, L. G. \& McDonald, L. C. Burden of Clostridium difficile

573

574

575

576

577

578

579

580

581

582

583

584

585

586

587

588

589

590

591

592

593

594

595

596

597

598

599

600

601

602

603

604

605

606

607

608

609

610

611

612

613

614

615

616

617

618

619 infection in the United States. The New England Journal of Medicine 372, 23692370, doi:10.1056/NEJMc1505190 (2015).

2 Hryckowian, A. J., Pruss, K. M. \& Sonnenburg, J. L. The emerging metabolic view of Clostridium difficile pathogenesis. Current Opinion in Microbiology 35, 4247, doi:10.1016/j.mib.2016.11.006 (2017).

$3 \quad$ Khanna, S. et al. The epidemiology of community-acquired Clostridium difficile infection: a population-based study. The American Journal of Gastroenterology 107, 89-95, doi:10.1038/ajg.2011.398 (2012).

$4 \mathrm{Ng}, \mathrm{K} . \mathrm{M}$. et al. Microbiota-liberated host sugars facilitate post-antibiotic expansion of enteric pathogens. Nature 502, 96-99, doi:10.1038/nature12503 (2013).

5 Ferreyra, J. A. et al. Gut Microbiota-Produced Succinate Promotes C. difficile Infection after Antibiotic Treatment or Motility Disturbance. Cell Host \& Microbe 6, 770-777, doi:10.1016/j.chom.2014.11.003 (2014).

6 Buffie, C. G. et al. Precision microbiome reconstitution restores bile acid mediated resistance to Clostridium difficile. Nature, doi:10.1038/nature13828 (2014).

$7 \quad$ Earle, K. A. et al. Quantitative Imaging of Gut Microbiota Spatial Organization. Cell Host \& Microbe 18, 478-488, doi:10.1016/j.chom.2015.09.002 (2015).

8 Hopkins, M. J. \& Macfarlane, G. T. Nondigestible oligosaccharides enhance bacterial colonization resistance against Clostridium difficile in vitro. Applied and Environmental Microbiology 69, 1920-1927 (2003).

9 Valdes-Varela, L., Hernandez-Barranco, A. M., Ruas-Madiedo, P. \& Gueimonde, M. Effect of Bifidobacterium upon Clostridium difficile Growth and Toxicity When Co-cultured in Different Prebiotic Substrates. Frontiers in Microbiology 7, 738, doi:10.3389/fmicb.2016.00738 (2016).

10 Kondepudi, K. K., Ambalam, P., Nilsson, I., Wadstrom, T. \& Ljungh, A. Prebioticnon-digestible oligosaccharides preference of probiotic bifidobacteria and antimicrobial activity against Clostridium difficile. Anaerobe 18, 489-497, doi:10.1016/j.anaerobe.2012.08.005 (2012).

11 Wlodarska, M., Willing, B. P., Bravo, D. M. \& Finlay, B. B. Phytonutrient diet supplementation promotes beneficial Clostridia species and intestinal mucus secretion resulting in protection against enteric infection. Scientific Reports 5, 9253, doi:10.1038/srep09253 (2015).

12 Moore, J. H. et al. Defined Nutrient Diets Alter Susceptibility to Clostridium difficile Associated Disease in a Murine Model. PLoS One 10, e0131829, doi:10.1371/journal.pone.0131829 (2015).

13 Kampmann, C., Dicksved, J., Engstrand, L. \& Rautelin, H. Composition of human faecal microbiota in resistance to Campylobacter infection. Clin Microbiol Infect 22, 61 e61-68, doi:10.1016/j.cmi.2015.09.004 (2016).

14 Ferreira, R. B. et al. The intestinal microbiota plays a role in Salmonella-induced colitis independent of pathogen colonization. PLoS One 6, e20338, doi:10.1371/journal.pone.0020338 (2011).

15 Seekatz, A. M. et al. Recovery of the gut microbiome following fecal microbiota transplantation. mBio 5, e00893-00814, doi:10.1128/mBio.00893-14 (2014).

16 Walker, A. W. et al. Dominant and diet-responsive groups of bacteria within the human colonic microbiota. The ISME journal 5, 220-230,

620 doi:10.1038/ismej.2010.118 (2011). 
62117 Lawley, T. D. et al. Targeted restoration of the intestinal microbiota with a simple,

622

623

624

625

626

627

628

629

630

631

632

633

634

635

636 defined bacteriotherapy resolves relapsing Clostridium difficile disease in mice. PLoS Pathogens 8, e1002995, doi:10.1371/journal.ppat.1002995 (2012).

18 Schubert, A. M. et al. Microbiome data distinguish patients with Clostridium difficile infection and non- $C$. difficile-associated diarrhea from healthy controls. mBio 5, e01021-01014, doi:10.1128/mBio.01021-14 (2014).

19 Schubert, A. M., Sinani, H. \& Schloss, P. D. Antibiotic-Induced Alterations of the Murine Gut Microbiota and Subsequent Effects on Colonization Resistance against Clostridium difficile. mBio 6, e00974, doi:10.1128/mBio.00974-15 (2015).

20 Rojo, D. et al. Clostridium difficile heterogeneously impacts intestinal community architecture but drives stable metabolome responses. The ISME Journal 9, 22062220, doi:10.1038/ismej.2015.32 (2015).

21 Lyras, D. et al. Toxin B is essential for virulence of Clostridium difficile. Nature 458, 1176-1179, doi:10.1038/nature07822 (2009).

22 Rolfe, R. D. Role of volatile fatty acids in colonization resistance to Clostridium difficile. Infection and Immunity 45, 185-191 (1984).

637

638

23 Karlsson, S., Lindberg, A., Norin, E., Burman, L. G. \& Akerlund, T. Toxins, butyric

639

640

641

642

643

644

645

646

647

648

649

650

651

652

653

654

655

656

657

658

659 acid, and other short-chain fatty acids are coordinately expressed and downregulated by cysteine in Clostridium difficile. Infection and Immunity 68, 58815888 (2000).

24 Kopke, M., Straub, M. \& Durre, P. Clostridium difficile is an autotrophic bacterial pathogen. PLoS One 8, e62157, doi:10.1371/journal.pone.0062157 (2013).

25 Martin-Verstraete, I., Peltier, J. \& Dupuy, B. The Regulatory Networks That Control Clostridium difficile Toxin Synthesis. Toxins 8, doi:10.3390/toxins8050153 (2016).

26 Kelly, C. J. et al. Crosstalk between Microbiota-Derived Short-Chain Fatty Acids and Intestinal Epithelial HIF Augments Tissue Barrier Function. Cell Host \& Microbe 17, 662-671, doi:10.1016/j.chom.2015.03.005 (2015).

27 Carmody, R. N. et al. Diet dominates host genotype in shaping the murine gut microbiota. Cell Host \& Microbe 17, 72-84, doi:10.1016/j.chom.2014.11.010 (2015).

28 Rabbani, G. H. et al. Green banana reduces clinical severity of childhood shigellosis: a double-blind, randomized, controlled clinical trial. The Pediatric Infectious Disease Journal 28, 420-425, doi:10.1097/INF.0b013e31819510b5 (2009).

29 Alvarez-Acosta, T. et al. Beneficial role of green plantain [Musa paradisiaca] in the management of persistent diarrhea: a prospective randomized trial. Journal of the American College of Nutrition 28, 169-176 (2009).

30 Desai, M. S. et al. A Dietary Fiber-Deprived Gut Microbiota Degrades the Colonic Mucus Barrier and Enhances Pathogen Susceptibility. Cell 167, 1339-1353

660

661

662

663

664

665

666

667

668 e1321, doi:10.1016/j.cell.2016.10.043 (2016).

31 Sebaihia, M. et al. The multidrug-resistant human pathogen Clostridium difficile has a highly mobile, mosaic genome. Nature Genetics 38, 779-786, doi:10.1038/ng1830 (2006).

32 Cartman, S. T. \& Minton, N. P. A mariner-based transposon system for in vivo random mutagenesis of Clostridium difficile. Applied and Environmental Microbiology 76, 1103-1109, doi:10.1128/aem.02525-09 (2010).

33 Kashyap, P. C. et al. Complex interactions among diet, gastrointestinal transit, and gut microbiota in humanized mice. Gastroenterology 144, 967-977,

669 doi:10.1053/j.gastro.2013.01.047 (2013). 
67134 Sonnenburg, E. D. et al. Diet-induced extinctions in the gut microbiota compound

672

673

674

675

676

677

678

679

680

681

682

683

684

685

686 over generations. Nature 529, 212-215, doi:10.1038/nature16504 (2016).

35 Turnbaugh, P. J. et al. The effect of diet on the human gut microbiome: a metagenomic analysis in humanized gnotobiotic mice. Science Translational Medicine 1, 6ra14, doi:10.1126/scitranslmed.3000322 (2009).

36 Etienne-Mesmin, L. et al. Toxin-positive Clostridium difficile latently infect mouse colonies and protect against highly pathogenic $C$. difficile. Gut, doi:10.1136/gutjnl-2016-313510 (2017).

37 Sonnenburg, E. D. et al. Specificity of polysaccharide use in intestinal bacteroides species determines diet-induced microbiota alterations. Cell, 141, 1241-52, doi:10.1016/j.cell.2010.05.005 (2010).

38 Bachmanov, A. A., Reed, D. R., Beauchamp, G. K. \& Tordoff, M. G. Food intake, water intake, and drinking spout side preference of 28 mouse strains. Behavior Genetics 32, 435-443 (2002).

39 Sampson, T. R. et al. Gut Microbiota Regulate Motor Deficits and

687

688

689 Neuroinflammation in a Model of Parkinson's Disease. Cell 167, 1469-

690

691

692

693

694

695

696

697

698

699

700

701

702

703 1480.e1412, doi:10.1016/j.cell.2016.11.018 (2016).

40 Gopinath, S., Lichtman, J. S., Bouley, D. M., Elias, J. E. \& Monack, D. M. Role of disease-associated tolerance in infectious superspreaders. Proceedings of the National Academy of Sciences of the United States of America 111, 1578015785, doi:10.1073/pnas.1409968111 (2014).

41 Caporaso, J. G. et al. Ultra-high-throughput microbial community analysis on the Illumina HiSeq and MiSeq platforms. The ISME journal 6, 1621-1624, doi:10.1038/ismej.2012.8 (2012).

42 Caporaso, J. G. et al. QIIME allows analysis of high-throughput community sequencing data. Nature Methods 7, 335-336, doi:10.1038/nmeth.f.303 (2010).

43 Edgar, R. C. Search and clustering orders of magnitude faster than BLAST. Bioinformatics (Oxford, England) 26, 2460-2461, doi:10.1093/bioinformatics/btq461 (2010).

44 Caporaso, J. G. et al. PyNAST: a flexible tool for aligning sequences to a template alignment. Bioinformatics (Oxford, England) 26, 266-267, doi:10.1093/bioinformatics/btp636 (2010).

45 McDonald, D. et al. An improved Greengenes taxonomy with explicit ranks for ecological and evolutionary analyses of bacteria and archaea. The ISME Journal 6, 610-618, doi:10.1038/ismej.2011.139 (2012).

705 